\title{
Brolucizumab: A Newly Developed Anti-VEGF Molecule for the Treatment of Neovascular Age-Related Macular Degeneration
}

\author{
Ramin Tadayoni $^{\mathrm{a}} \quad$ Laura Sararols $^{\mathrm{b}}$ Georges Weissgerber ${ }^{\mathrm{c}}$ Rohini Verma $^{\mathrm{c}}$ \\ Andreas Clemens ${ }^{c, d}$ Frank G. Holz ${ }^{\mathrm{e}}$ \\ a Université de Paris, Ophthalmology Department, AP-HP, Hôpital Lariboisière, Paris, France; ${ }^{b}$ Hospital General de \\ Catalunya, Barcelona, Spain; ' Novartis Pharma AG, Basel, Switzerland; d Department of Cardiology and Angiology I,

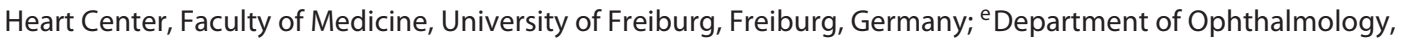 \\ University of Bonn, Bonn, Germany
}

\section{Keywords}

Brolucizumab · Anti-VEGF · Neovascular age-related macular degeneration (AMD) · Single-chain antibody fragment

\begin{abstract}
Background: Despite the success of anti-vascular endothelial growth factors (anti-VEGFs), currently, there is a need for highly effective compounds that can alleviate the burden of managing neovascular age-related macular degeneration (nAMD). Purpose: To review the milestones in the molecular and clinical development of brolucizumab, the first singlechain antibody fragment (scFv) designed specifically for intraocular use in humans. Methods: In this article, we summarize the preclinical and current clinical evidence of brolucizumab administration with an overview of the other treatment regimens and additional indications under investigation. Results: The unique molecular design of brolucizumab led to a low molecular weight of only $26 \mathrm{kDa}$, allowing for a concentrated molar dose of 1 intravitreal injection compared with other anti-VEGF agents. Phase I and II clinical trial outcomes validated the efficacy of brolucizumab in the treatment of $\mathrm{nAMD}$ with signals of a more durable treatment effect. The pivotal phase III trials, HAWK and HARRIER, which
\end{abstract}

included a total of 1,817 patients, established that brolucizumab can be administered every 3 months while maintaining disease control. Conclusions: The preclinical and clinical data on brolucizumab provide evidence of sustained disease control with longer injection intervals, thus potentially reducing the treatment burden in patients with nAMD.

(C) 2021 S. Karger AG, Base

\section{Introduction}

\section{Overview}

Almost a decade since the first approval of an antivascular endothelial growth factor (VEGF) for intravitreal treatment of neovascular age-related macular degeneration (nAMD), brolucizumab marks an important milestone in the field of ophthalmology. Its development represents a molecular breakthrough by overcoming the technological challenges associated with the design of single-chain antibody fragments (scFvs) for therapeutic application $[1,2]$. The molecular characteristics demonstrated by brolucizumab in preclinical and clinical trials

A.C. and F.G.H. contributed equally to this work. karger@karger.com

www.karger.com/oph

Karger ${ }^{\prime}=$
(C) 2021 S. Karger AG, Basel

CH-4056 Basel (Switzerland)
andreas.clemens @ novartis.com
Andreas Clemens

Novartis Pharma AG 
can help address the challenges associated with current anti-VEGF therapies, such as: the need for higher dosing; loss of efficacy over time due to undertreatment; and treatment burden, fatigue, and discontinuation with subsequent visual loss $[3,4]$.

In a first-in-human trial, brolucizumab showed longer durability of effect by delaying the need for rescue medication when compared with the anti-VEGF ranibizumab [5]. The phase II and III noninferiority trials of brolucizumab versus aflibercept revealed comparable gains in visual outcomes that were sustained over time, and superior anatomical outcomes and fluid resolution $[6,7]$. Data from these studies have been submitted to health authorities worldwide. The first approval of the treatment for patients with nAMD was obtained in October 2019 in the USA, with further approval of this indication being granted in Japan, Australia, Switzerland, and the European Union.

This article describes the innovative approach to the development of brolucizumab, its salient molecular characteristics and clinical profile, and the added value to the anti-VEGF treatment armamentarium in nAMD.

\section{Unmet Needs in the Treatment of $n A M D$}

nAMD is a chronic and progressive neurodegenerative disease characterized by macular atrophy, scarring, and chronic exudation. Intravitreal anti-VEGF agents have marked an important milestone in the treatment of this condition. The licensed anti-VEGF drugs, aflibercept and ranibizumab, act by inhibiting choroidal neovascular $(\mathrm{CNV})$-associated vessel growth and capillary leakage in nAMD [8]. This mechanism of action has led to a dramatic 50\% reduction in the incidence of blindness over the 12 years of anti-VEGF use [9]. However, real-world data, such as those from the LUMINOUS observational studies of ranibizumab, have shown that many patients do not receive the full loading dose and that this is one of the reasons for suboptimal visual outcomes [10]. Moreover, the requirement for maintenance with repeated intravitreal injections of the available anti-VEGFs and regular visits to the clinic poses a burden to clinicians, patients, and the healthcare system [11].

The ultimate goal of treatment in nAMD is to reduce disease activity to a minimum [12]. As fluid within different layers of the retina is a pathomorphological correlate of disease activity, fluid resolution is another key consideration when evaluating the potency of emerging therapies. These aspects are of high relevance as they would translate into a longer duration of therapeutic action and thus improve patient care and outcomes in nAMD.

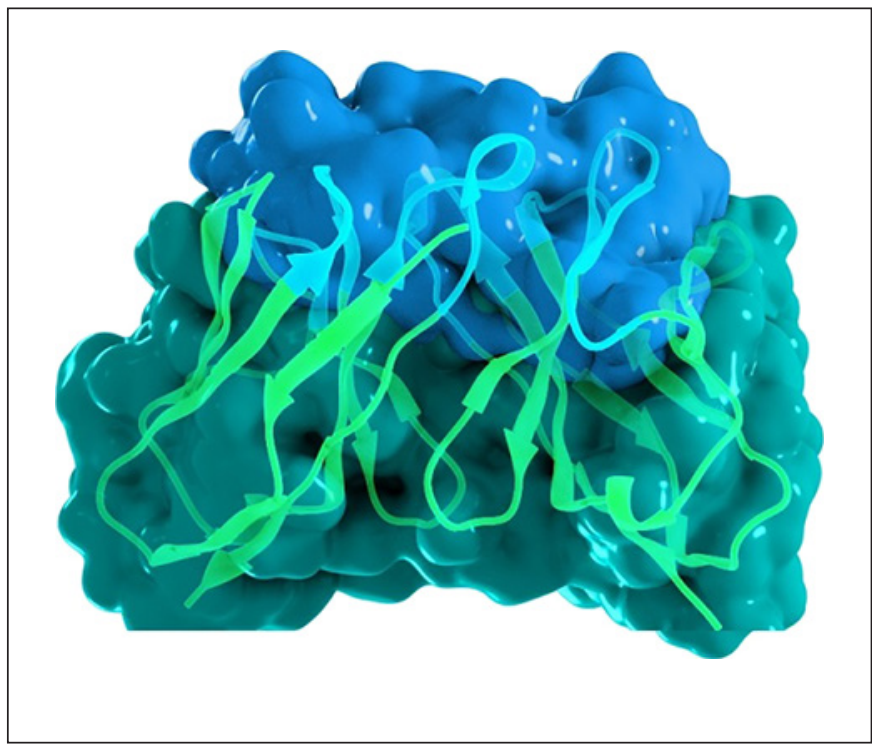

Fig. 1. The molecular structure of brolucizumab.

These conclusions were further strengthened by the results of a post hoc analysis of the CATT and IVAN trials, in which stabilization (lower fluctuation) of central subfield thickness (CST) translated into better visual acuity and lower rates of fibrosis and geographic atrophy [13].

Aside from anti-VEGFs, other innovative approaches to treatment or delivery, e.g., gene therapy or the development of long-acting drug delivery systems, also hold promise for the management of this disease $[8,14]$.

\section{Brolucizumab: A Molecular Breakthrough}

\section{The Generation of a scFv for Intraocular Use}

The scFvs are autonomous agents that do not depend on a heavy molecular support structure but retain full binding capacity to the target, thus providing a concentrated, highly effective molecule [15]. Brolucizumab is comprised of the variable domains of the monoclonal antibody $(\mathrm{mAb})$, joined by a short flexible linker peptide which provides the required stability (Fig. 1). The absence of the $\mathrm{Fc}$ region and the small molecular size of scFvs are advantageous from a pharmacokinetic as well as a manufacturing standpoint [15]. The $\mathrm{V}_{\mathrm{H}}$ and $\mathrm{V}_{\mathrm{L}}$ variable domain chains of brolucizumab are genetically linked; thus, unlike the full $\mathrm{mAb}$, the molecule can be expressed from a single genetic transcript [16].
Tadayoni/Sararols/Weissgerber/Verma/ Clemens/Holz 


\begin{tabular}{|c|c|c|c|c|}
\hline & Aflibercept & Ranibizumab & $\begin{array}{c}\begin{array}{c}\text { Unlicensed } \\
\text { bevacizumab }\end{array} \\
\end{array}$ & Brolucizumab \\
\hline Format & $\begin{array}{l}\text { VEGFR } 1 / 2-F c \text { fusion } \\
\text { protein }\end{array}$ & Fab fragment & Full antibody (lgG1) & $\begin{array}{c}\text { Single-chain } \\
\text { antibody fragment }\end{array}$ \\
\hline \multicolumn{5}{|l|}{ Molecular structure } \\
\hline Molecular weight & $97-115 \mathrm{kDa}$ & $\sim 48 \mathrm{kDa}$ & $\sim 149 \mathrm{kDa}$ & $26 \mathrm{kDa}$ \\
\hline Clinical dose & $2.00 \mathrm{mg}$ & $0.50 \mathrm{mg}$ & $1.25 \mathrm{mg}$ & $6.00 \mathrm{mg}$ \\
\hline $\begin{array}{l}\text { Relative number of } \\
\text { molecules per } \\
\text { injection }\end{array}$ & 1.0 & $0.5-0.6$ & $0.4-0.5$ & $11.2-13.3$ \\
\hline
\end{tabular}

Fig. 2. Molecular characteristics and clinical dosing of brolucizumab by comparison with other anti-VEGF agents $\mathrm{CH}$, constant heavy; CL, constant light; Fab, fragment, antigen-binding; Fc, fragment crystallizable; IgG, immunoglobulin G; VEGFR, vascular endothelial growth factor receptor; VH, variable heavy; VL, variable light.

Brolucizumab was isolated by rabbit immunization and humanized by grafting the complementarity-determining regions (CDRs) onto a highly stable, proprietary human antibody framework [2]. By combining stabilization and humanization in 1 step, this procedure is particularly attractive for therapeutic applications [1]. Indeed, grafting of the CDRs onto the stable human framework was found to produce a molecule with remarkable thermal stability and solubility, usually a challenge to achieve with scFvs [1]. The marked structural differences between brolucizumab and other VEGF inhibitors in ophthalmology established brolucizumab as a next-generation anti-VEGF available for therapeutic use.

\section{Molecular Characteristics}

The molecular properties of brolucizumab have been characterized in vitro and compared with those of aflibercept, ranibizumab, and bevacizumab under standardized conditions $[17,18]$. The preclinical studies of brolucizumab were conducted on rabbits and cynomolgus monkeys [18-20], similar to other anti-VEGF agents $[21,22]$.

Intravitreal ranibizumab has also been studied in a laser-induced model of CNV in monkeys [23]. Of note, comparisons between molecules are relevant only if conducted in the same species and using identical assays un-

Brolucizumab for the Treatment of Neovascular AMD der standard conditions; this may explain some of the discrepancies in the data on molecular characteristics published so far.

\section{Binding Affinity to VEGF-A}

Significantly higher binding affinity to VEGF-A isoforms was observed for brolucizumab when compared with the oncology drug bevacizumab [2]. Brolucizumab bound with high affinity to all VEGF-A isoforms, with a similar, but numerically higher affinity than aflibercept or ranibizumab when investigated in the same assay and under identical conditions [18]. Brolucizumab also exhibited a 42 - to 54-fold faster on-rate than ranibizumab; this translated into different in vitro potencies, with brolucizumab blocking binding to VEGFR2 at approximately 25to 50-fold lower concentrations than ranibizumab [18].

\section{Molar Dosing}

Owing to its molecular design as a scFv, brolucizumab is currently the VEGF-binding compound with the lowest molecular weight (26 kDa [20]; Fig. 2). It binds to VEGF-A in a 2:1 ratio; this is reduced to $1: 1$ as the concentration decreases over time, but full blockage of VGEFA is still present at this binding ratio.

The low molecular weight of brolucizumab $(4 \times$ lower than that of aflibercept and $1.8 \times$ lower than ranibizumab) 
Table 1. Pharmacokinetic characteristics and clinical outcomes (anatomical and functional) observed with brolucizumab in comparison to currently approved anti-VEGFs

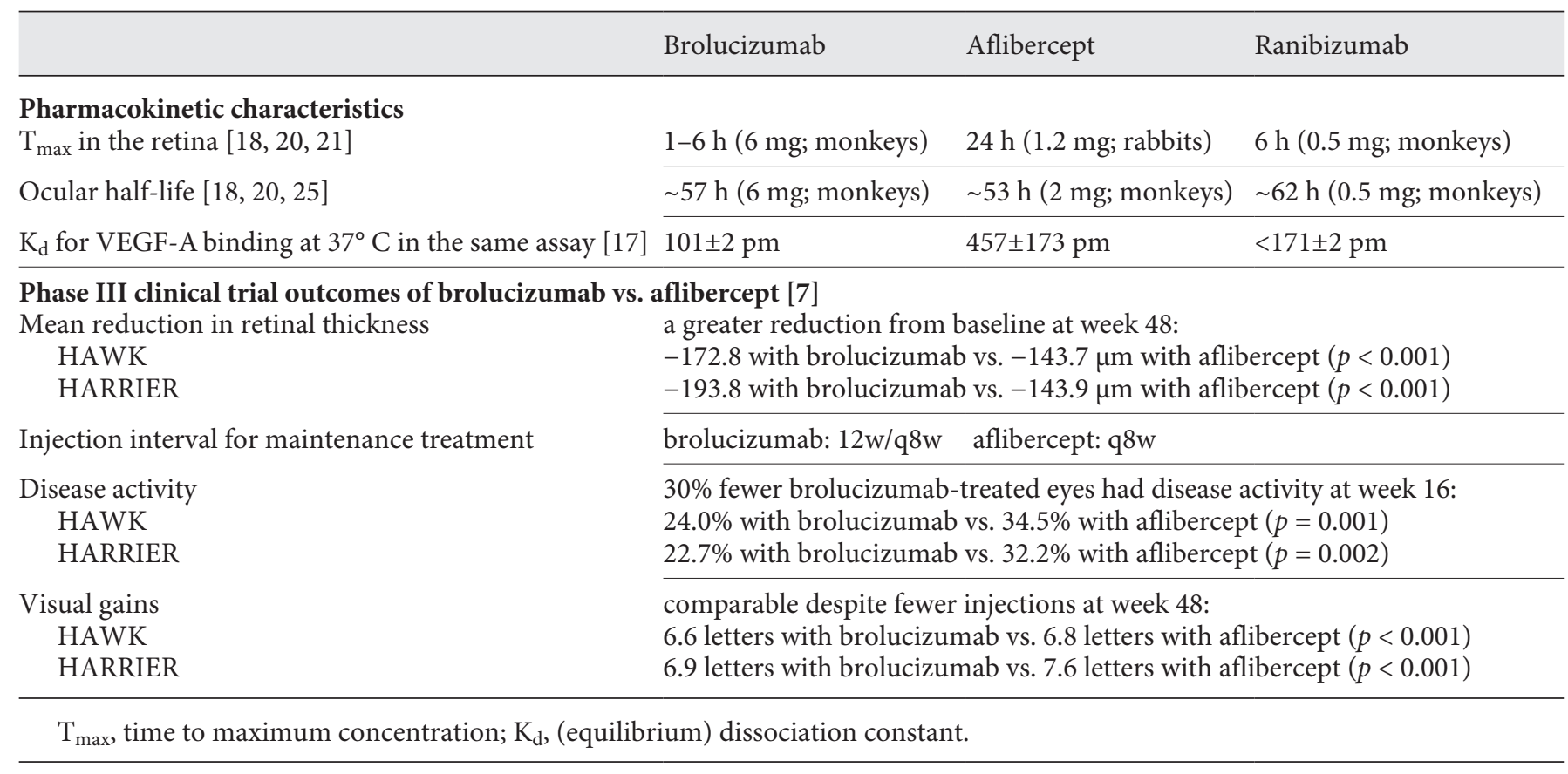

permits a molar dosing around 12-fold higher than with aflibercept and 22-fold higher than with ranibizumab, respectively (Fig. 2) [17, 24]. Clinical doses of up to $6 \mathrm{mg}$ of brolucizumab can thus be delivered in a single $0.05 \mathrm{~mL}$ intravitreal injection (Fig. 2).

Penetration of the Retina and Choroid

The smaller molecular size of brolucizumab provides for potentially more effective penetration of the retina and choroid than other anti-VEGF molecules [24]. In rabbits, brolucizumab showed a 2.2-fold higher exposure in the retina and a 1.7-fold higher exposure in RPE/choroid than ranibizumab [18].

The PK profile after intravitreal injections of 1 and 6 mg of brolucizumab in cynomolgus monkeys was similar to that observed in rabbits, with high exposures in the retina and RPE/choroid. The retinal exposure of brolucizumab in cynomolgus monkeys was 3.1-fold higher than that reported for ranibizumab. Meanwhile, the half-life of the 2 molecules in the retinal tissue were not significantly different [18].

The time to maximum concentration in the retina was 1-6 h for brolucizumab [19], compared with $6 \mathrm{~h}$ for ranibizumab in monkeys [22] and $24 \mathrm{~h}$ for aflibercept in a rabbit model [21, 25] (Table 1).

\section{Systemic Clearance}

Brolucizumab exhibits a rapid systemic clearance $(5.6 \pm 1.5 \mathrm{~h})$, which is an essential prerequisite for a lower probability of systemic side effects $[19,20]$, while retinal exposure remains high (Fig. 3). In cynomolgus monkeys, maximal serum concentrations after intravitreal injection of brolucizumab (6 mg) were $>6,000$-fold lower than those in the vitreous [19]. In a preclinical study of ranibizumab in the same animal model, the overall drug concentration after 1 intravitreal injection was 1,500-fold lower than that in the vitreous, and the peak concentration of ranibizumab in the retina was approximately onethird of that in the vitreous [22].

Brolucizumab was cleared from the ocular compartments with a mean terminal half-life of $56.8 \pm 7.6 \mathrm{~h}$ in monkeys [19]. For comparison, ranibizumab was cleared from all ocular compartments with an ocular half-life of approximately $62 \mathrm{~h}$ [22], and aflibercept cleared with a half-life of $53 \mathrm{~h}$ in the same model [26] (Table 1).

The rapid systemic clearance of brolucizumab is presumed to be due to its small molecular size and the absence of a Fc domain. Unlike full-length antibodies (i.e., aflibercept), which are subject to a conservation mechanism, molecules without a Fc region are targeted for degradation and have no cumulative effect even after multiple injections [27].
Tadayoni/Sararols/Weissgerber/Verma/ Clemens/Holz 


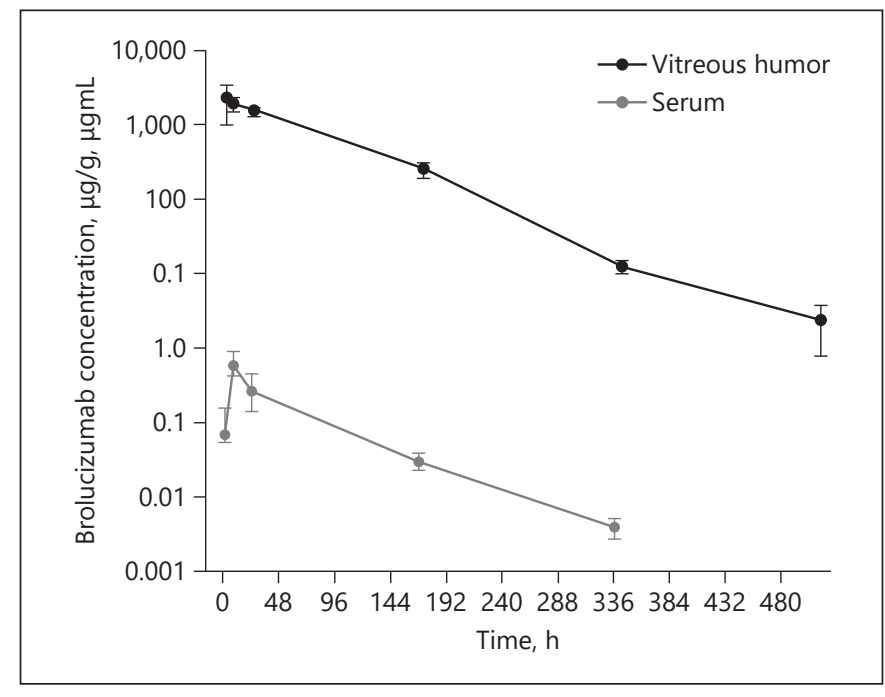

Fig. 3. Systemic clearance of brolucizumab: free drug concentrations following intravitreal injection in cynomolgus monkeys.

\section{Clinical Evidence of Brolucizumab Use}

Two reviews on brolucizumab and the visual, anatomic, and safety outcomes from the key clinical trials were recently published $[14,28,29]$. Here, we summarize the main milestones in the clinical development of brolucizumab, outlining the rationale and role of these key studies, and briefly conclude with the Phase IIIa and b clinical outlook for this agent.

\section{The Phase I/II SEE Study: First-in-Human Trial}

The multicenter, double-masked, randomized, 6month, ascending single-dose SEE study (NCT01304693) assessed the safety and efficacy of brolucizumab vs ranibizumab in 194 treatment-naïve nAMD patients [5]. The study demonstrated noninferiority to ranibizumab in the mean CST change after a single intravitreal injection of brolucizumab (4.5 or $6.0 \mathrm{mg}$ ). A dose-dependent effect was also observed for brolucizumab in this trial [5]. The 30-day delay in time to retreatment after 1 injection highlighted the longer durability of the effect of brolucizumab than ranibizumab. This allowed for the design of a maintenance treatment arm with brolucizumab injections being administered every 12 weeks ( 3 months) in the phase III trials to address the treatment frequency burden.

\section{The Phase II OSPREY Study: A Long-Term,}

Head-to-Head Trial of Brolucizumab versus Aflibercept

OSPREY (NCT01796964) was a prospective, randomized, double-masked, multicenter study conducted on 89 treatment-naïve patients to compare the efficacy and safety of repeated doses of brolucizumab $6.0 \mathrm{mg}$ and aflibercept $2.0 \mathrm{mg}$ [6]. OSPREY included a 40-week, head-to-head comparison of brolucizumab and aflibercept.

The study met its primary end point at weeks 12 and 16 , demonstrating the noninferiority of brolucizumab to aflibercept, with respect to visual acuity gains. $\mathrm{Pa}$ tients in the brolucizumab and aflibercept groups also showed comparable gains in best corrected visual acuity (BCVA) from baseline to week 40 in the matched 8-week (q8w) treatment phase. At week 40, the BCVA change from baseline was +6.3 Early Treatment Diabetic Retinopathy Study (ETDRS) letters for brolucizumab and +5.8 ETDRS letters for aflibercept, a treatment difference of 0.5 ETDRS letters (80\%; 95\% CI -3.39 to 4.39 ). The brolucizumab group had more stable CST reductions, higher rates of fluid resolution, and fewer unscheduled treatments than the aflibercept group. Overall, around half of the patients receiving treatment in the unmasked brolucizumab 12-week ( $12 \mathrm{w})$ phase after week 40 maintained visual acuity without any additional rescue therapy [6].

The results of the OSPREY trial informed the final design of the phase III studies. The anatomical superiority demonstrated in OSPREY was subsequently replicated in the phase III trials, and a signal of effective q12w maintenance treatment with brolucizumab was also replicated.

\section{Phase III HAWK and HARRIER Trials}

The two 96-week, prospective, double-masked, multicentered studies, HAWK (NCT02307682) and HARRIER (NCT02434328), investigated the efficacy and safety of brolucizumab versus aflibercept in nAMD patients [7]. In these 2 trials, patients on brolucizumab were treated with injections at weeks 0,4 , and 8 (loading phase), and then every 12 weeks (q12w) unless disease activity was identified. In this event, a permanent adjustment to a treatment interval of every 8 weeks ( $q 8 \mathrm{w}$ ) was applied. To rapidly identify the patients with a higher anti-VEGF treatment need after loading, the protocol guidance at week 16 provided specific functional and anatomical criteria for CST and intraretinal fluid (IRF) status assessed by spectral domain OCT (i.e., a decrease in BCVA of $\geq 5$ ETDRS letters vs. baseline, a decrease in BCVA of $\geq 3$ ETDRS letters and a CST increase $\geq 75 \mu \mathrm{m}$ vs. week 12 , a decrease in BCVA of $\geq 5$ ETDRS letters due to nAMD disease activity vs. week 12 , or the appearance of new or worse intraretinal cysts/ 
IRF vs. week 12). Thereafter, the dosing guidance was based on BCVA decline due to nAMD activity compared with week 12 (i.e., a decrease in BCVA of $\geq 5$ ETDRS letters due to nAMD disease activity vs. week 12). Ultimately, the masked investigator made the final treatment decisions based on clinical judgement. The results were collectively reported as a $\mathrm{q} 12 \mathrm{w} / \mathrm{q} 8 \mathrm{w}$ regimen and compared to a $\mathrm{q} 8 \mathrm{w}$ dosing ( $3 \times$ monthly loading) of aflibercept [7].

The primary end point of both HAWK and HARRIER was noninferiority of brolucizumab to aflibercept in mean BCVA change from baseline to week 48 . A key secondary end point was the $\mathrm{q} 12 \mathrm{w}$ treatment status at week 48; additional secondary efficacy end points included changes in BCVA and CST at each visit after baseline, IRF and/or subretinal fluid (SRF) status, and detectable disease activity at week 16 .

The pivotal trials achieved their primary end point, with BCVA gains from baseline of +6.6 and +6.8 ETDRS letters (HAWK) and +6.9 and +7.6 ETDRS letters (HARRIER) with brolucizumab and aflibercept, respectively (Table 1). These vision gains were sustained up to week 96 in an analysis of the 2-year outcomes from the pivotal trials [30].

The superior reductions in CST from baseline observed with brolucizumab at week 16 were maintained at week 48 [7] and 96 [30], despite less frequent injections in the brolucizumab group. This is an important result, as variation in retinal thickness during anti-VEGF treatment was found to be associated with worse BCVA outcomes and the development of fibrosis in a recent analysis [13].

Regarding $\mathrm{q} 12 \mathrm{w}$ treatment status at week 48, 56\% (HAWK) and 51\% (HARRIER) of the patients receiving brolucizumab $6 \mathrm{mg}$ were treated constantly every 3 months. Regarding the end point of disease activity, brolucizumab $(6 \mathrm{mg})$ showed superiority over aflibercept, with $30 \%$ less disease activity (after loading) at week 16 (Table 1) [7].

In the absence of a placebo-controlled trial, there is a need to assess the clinical value of brolucizumab relative to the natural course of nAMD. A comparison of treatment effect data in patients receiving brolucizumab $6 \mathrm{mg}$ in the HAWK and HARRIER trials with putative (modeled) placebo data showed that brolucizumab was associated with an overall BCVA gain of approximately 22 ETDRS letters by week 48 and 28 ETDRS letters by week 96 , thereby underscoring the clinical value of this novel antiVEGF agent [31].

\section{Fluid Resolution in the Brolucizumab Trials}

The presence of retinal fluid on OCT is an important indicator of disease activity [11]. In the OSPREY study, both brolucizumab and aflibercept were given with the same treatment frequency (q8w) until week 40, allowing for a direct comparison of their treatment effects. A post hoc analysis of OSPREY found that a greater proportion of brolucizumab-treated eyes (61\% vs. $35 \%$ for aflibercept, i.e., a difference of $26.0 \%$ [ $80 \%$; 95\% CI 12.2-39.7]) achieved simultaneous resolution of IRF and SRF at week 40 [6]. Additionally, higher percentages of patients had consecutive fluid-free visits with brolucizumab than aflibercept at week 40 (75.00 and $46.67 \%$ patients with $\geq 2$ consecutive visits, respectively, and 68.18 and $37.78 \%$ with $\geq 3$ consecutive visits, respectively) [32].

In both HAWK and HARRIER, fewer patients receiving brolucizumab $6 \mathrm{mg}$ vs. aflibercept had IRF and/or SRF at week 16 (35\% in HAWK and 36\% in HARRIER; $p<0.001$ for both) and week 48 (31\% in HAWK, $41 \%$ in HARRIER; $p<0.001$ for both). Similarly, $\geq 30 \%$ fewer patients treated with brolucizumab than those treated with aflibercept had sub-RPE fluid at week 16 and 48 in both trials [7]. The proportions of eyes with IRF/SRF at week 96 in HAWK were 24\% for brolucizumab $6 \mathrm{mg}$ and 37\% for aflibercept $(p=0.0002)$; in HARRIER, they were $24 \%$ for brolucizumab $6 \mathrm{mg}$ and 39\% for aflibercept $(p<$ 0.0001) [30].

Taken together, the analyses of the phase II and III trials indicate that brolucizumab provides better fluid control than aflibercept in all retina compartments, both in the head-to-head comparison to aflibercept with identical dosing frequencies ( $\mathrm{q} 8 \mathrm{w}$ ) and when given at a lower frequency (q12/q8).

\section{Brolucizumab and Durability of Effect}

The prerequisite for longer durability with an antiVEGF agent is potent fluid resolution, which has been demonstrated by brolucizumab. Consequently, in the first-in-human SEE trial, the median time until requirement for another injection was found to be 30 days longer with brolucizumab at the 3 and $6 \mathrm{mg}$ doses and 15 days longer at the $4.5 \mathrm{mg}$ dose, compared with ranibizumab, indicating a more durable therapeutic effect [5].

The phase II OSPREY trial provided further support for durability of action, with twice as many patients treated with brolucizumab than those treated with aflibercept receiving unscheduled additional treatment ("rescue therapy") up to week 40. Furthermore, approximately $50 \%$ of the brolucizumab-treated eyes had stable BCVA during q12 cycles in this study (until week 56) [6].
98

Ophthalmologica 2021;244:93-101 DOI: $10.1159 / 000513048$
Tadayoni/Sararols/Weissgerber/Verma/ Clemens/Holz 
The HAWK and HARRIER pivotal trials confirmed the highly consistent therapeutic efficacy and maintenance of effect observed in the phase I/II studies of brolucizumab. More than $50 \%$ of patients treated with brolucizumab $6 \mathrm{mg}$ in HAWK and HARRIER were maintained on a q12w injection interval immediately after the loading phase up to week 48 [7].

Over $75 \%$ of the brolucizumab 6 mg-treated eyes completing week 48 on a q12w injection interval continued successfully until week 96 (i.e., they showed no disease activity at the last assessment of this at week 92), thus qualifying for an eighth consecutive $\mathrm{q} 12 \mathrm{w}$ treatment interval [30].

\section{Safety Profile of Brolucizumab}

Overall, the incidence of adverse events (AEs) observed in the phase I-III clinical trials of brolucizumab was consistent with other published data on anti-VEGF treatment, thereby indicating comparable safety and tolerability profiles among these agents. In HAWK and HARRIER (48 weeks), the ocular and nonocular side effects were similar in the brolucizumab and aflibercept arms $[7,30]$.

In HAWK, AEs of interest included uveitis and iritis (2.2\% for each) with rates for brolucizumab $6 \mathrm{mg}$ and aflibercept of 0.3 and $0 \%$, respectively; the corresponding incidence was $<1 \%$ in both arms in HARRIER. An imbalance of very low numbers of uveitis serious AEs (SAEs) between brolucizumab and aflibercept was observed in both trials, and an imbalance of very low numbers of endophthalmitis SAEs was observed in HAWK, but was not confirmed in HARRIER. The overall number of SAEs was low in both trials $[7,30]$.

In the postmarketing usage of brolucizumab, there were reports of cases with intraocular inflammation and retinal occlusive vasculitis, with and without severe loss of vision. A thorough review of the reported cases and evidence from pivotal trials was conducted in a collaborative exercise involving the reporting physicians, retina specialists, an external safety review committee, and the marketing authorization holder. Novartis concluded that there was a confirmed safety signal of AEs termed as "retinal vasculitis and/or retinal vascular occlusion" that may result in a severe loss of vision. Typically, these events occurred in the presence of intraocular inflammation. As the review process is still ongoing at the time of this publication, updated information is being provided to the medical community by Novartis on a regular basis. More details can be found on the website of the marketing authorization holder: www.brolucizumab.info. Of note, an expert opinion on the management of intraocular inflammation, retinal vasculitis, and/or vascular occlusion was recently published [33].

\section{Phase III a and $b$ Clinical Outlook}

While the phase III results have demonstrated the effectiveness and durability of brolucizumab in a fixeddose regimen, there is interest in exploring its efficacy and durability with individualized treat-and-extend strategies, which are reflective of the current routine clinical practice globally [14].

Several phase IIIa and b studies are planned or have already started, aiming to address some of the unanswered questions not addressed in the phase III trials. Among these, the TALON trial (NCT04005352) has a superiority design and aims to demonstrate that brolucizumab allows for longer injection intervals than aflibercept in a treat-to-control regimen in treatment-naïve nAMD patients. TALON is a 64 -week, 2 -arm, randomized, double-masked, multicenter study, and it started recruiting in September 2019.

The phase I-III trials have not assessed brolucizumab as a dosing regimen of more often than every 8 weeks, outside of the initial 3-month loading phase. However, it is known from clinical trials and from experience with other anti-VEGF agents that some patients need more intensive (i.e., monthly) treatments. The ongoing phase IIIa MERLIN study (NCT03710564) is evaluating the safety and efficacy of monthly brolucizumab compared to monthly aflibercept in pretreated patients with persistent retinal fluid. MERLIN is a randomized, double-masked study being conducted across 50-65 sites in the USA. It will evaluate the performance of brolucizumab in difficult-to-treat patients after a switch from active aflibercept treatment, and assess the efficacy and safety profile of both drugs in this patient population.

Brolucizumab is also being investigated in other antiVEGF-responsive indications, such as diabetic macular edema (in the BUZZARD study [NCT04079231] and the noninferiority studies KITE [NCT03481660], KESTREL [NCT03481634], and KINGFISHER [NCT03917472]), andretinalveinocclusion(intheRAPTOR[NCT03802630] and RAVEN [NCT03810313] studies).

\section{Conclusions}

The development of brolucizumab has resulted in a highly effective therapeutic molecule with concentrated molar dosing that shows robust gains in visual acuity, su- 
perior fluid resolution, and longer durability of effect than other anti-VEGFs approved for the treatment of patients with nAMD [5-7]. Furthermore, the OSPREY trial and HAWK and HARRIER pivotal trials provide evidence that brolucizumab can reduce treatment burden by allowing for longer injection intervals with sustained disease control $[7,30]$. This is expected to improve longterm outcomes by reducing treatment burden and increasing patient adherence as well as helping to manage undercontrolled patients with the currently available anti-VEGFs.

More evidence on the clinical efficacy of brolucizumab and its impact on clinical practice in other anti-VEGFresponsive indications is expected in the coming years, highlighting the potential utility of this compound beyond nAMD.

\section{Acknowledgements}

Writing assistance in the preparation of this article was provided by Ileana Stoica, $\mathrm{PhD}$, of Novartis Ireland Ltd. (support for this assistance was funded by Novartis).

\section{Conflict of Interest Statement}

R.T. is a consultant for Alcon, Allergan, Bayer, B + L, Genentech, Moria, Novartis, Oculis, Roche, Thea, and Zeiss. A.C., G.W., and R.V. are full time employees and shareholders at Novartis Pharma AG. F.G.H. is a consultant for and/or has received funding for research from: Acucela, Allergan, Apellis, Bayer, BoehringerIngelheim, Bioeq/Formycon, CenterVue, Ellex, Roche/Genentech, Geuder, Grayburg Vision, Heidelberg Engineering, Kanghong, LinBioscience, NightStarX, Novartis, Optos, Pixium Vision, Oxurion, Stealth BioTherapeutic, and Zeiss.

\section{Funding Sources}

The sponsorship of this study and article processing charges were funded by Novartis Pharma AG, Basel, Switzerland.

\section{Author Contributions}

All authors had full access to all data in this study and take complete responsibility for the integrity of the data and accuracy of the data analysis. All named authors meet the International Committee of Medical Journal Editors (ICMJE) criteria for authorship for this article, take responsibility for the integrity of the work as a whole and gave their approval for this version to be published.

\section{References}

1 Borras L, Gunde T, Tietz J, Bauer U, Hulmann-Cottier V, Grimshaw JP, et al. Generic approach for the generation of stable humanized single-chain Fv fragments from rabbit monoclonal antibodies. J Biol Chem. 2010 Mar;285(12):9054-66.

2 Szabó E, Phillips DJ, Droste M, Marti A, Kretzschmar T, Shamshiev A, et al. Antitumor activity of DLX1008, an anti-VEGFA antibody fragment with low picomolar affinity, in human glioma models. J Pharmacol Exp Ther. 2018 May;365(2):422-9.

3 Holz FG, Tadayoni R, Beatty S, Berger A, Cereda MG, Cortez R, et al. Multi-country real-life experience of anti-vascular endothelial growth factor therapy for wet age-related macular degeneration. Br J Ophthalmol. 2015 Feb;99(2):220-6.

4 Vaze A, Fraser-Bell S, Gillies M. Reasons for discontinuation of intravitreal vascular endothelial growth factor inhibitors in neovascular age-related macular degeneration. Retina. 2014 Sep;34(9):1774-8.

5 Holz FG, Dugel PU, Weissgerber G, Hamilton R, Silva R, Bandello F, et al. Single-chain antibody fragment VEGF inhibitor RTH258 for neovascular age-related macular degeneration: A randomized controlled study. Ophthalmology. 2016 May;123(5):1080-9.

6 Dugel PU, Jaffe GJ, Sallstig P, Warburton J, Weichselberger A, Wieland M, et al. Broluci- zumab versus aflibercept in participants with neovascular age-related macular degeneration: A randomized trial. Ophthalmology. 2017 Sep;124(9):1296-304.

7 Dugel PU, Koh A, Ogura Y, Jaffe GJ, SchmidtErfurth U, Brown DM, et al.; HAWK and HARRIER Study Investigators. HAWK and HARRIER: Phase 3, multicenter, randomized, double-masked trials of brolucizumab for neovascular age-related macular degeneration. Ophthalmology. 2020 Jan;127(1):7284.

8 Holz FG, Schmitz-Valckenberg S, Fleckenstein M. Recent developments in the treatment of age-related macular degeneration. J Clin Invest. 2014 Apr;124(4):1430-8.

9 Bloch SB, Larsen M, Munch IC. Incidence of legal blindness from age-related macular degeneration in Denmark: year 2000 to 2010. Am J Ophthalmol. 2012 Feb;153(2):209-213. e2.

10 Holz FG. Treatment patterns in prior ranibizumab-treated patients with neovascular agerelated macular degeneration: real-world outcomes from the LUMINOUS study. Presented at EURETINA; 2017.

11 Schmidt-Erfurth U, Chong V, Loewenstein A, Larsen M, Souied E, Schlingemann R, et al.; European Society of Retina Specialists. Guidelines for the management of neovascular age-related macular degeneration by the
European Society of Retina Specialists (EURETINA). Br J Ophthalmol. 2014 Sep; 98(9):1144-67.

12 Arnold JJ, Markey CM, Kurstjens NP, Guymer RH. The role of sub-retinal fluid in determining treatment outcomes in patients with neovascular age-related macular degeneration-a phase IV randomised clinical trial with ranibizumab: the FLUID study. BMC Ophthalmol. 2016 Mar;16(1):31.

13 Evans RN, Reeves BC, Maguire MG, Martin DF, Muldrew A, Peto T, et al. Associations of variation in retinal thickness with visual acuity and anatomic outcomes in eyes with neovascular age-related macular degeneration lesions treated with anti-vascular endothelial growth factor agents. JAMA Ophthalmol. 2020 Oct;138(10):1043-51.

14 Yannuzzi NA, Freund KB. Brolucizumab: evidence to date in the treatment of neovascular age-related macular degeneration. Clin Ophthalmol. 2019 Jul;13:1323-9.

15 Miller BR, Demarest SJ, Lugovskoy A, Huang F, Wu X, Snyder WB, et al. Stability engineering of scFvs for the development of bispecific and multivalent antibodies. Protein Eng Des Sel. 2010 Jul;23(7):549-57.

16 Sharma A, Kumar N, Kuppermann BD, Bandello F. Brolucizimab-leading an era of structural revolution for long-term VEGF suppression. Eye (Lond). 2020 Apr;34(4):611-3. 
17 Tietz J, Spohn G, Schmid G, Konrad J, Jampen $\mathrm{S}$, Maurer $\mathrm{P}$, et al. Affinity and potency of RTH258 (ESBA1008), a novel inhibitor of vascular endothelial growth factor A for the treatment of retinal disorders. Invest Ophthalmol Vis Sci. 2015;56 (Abstract 150).

18 Borras L, Lenherr-Frey D, Grimshaw J, Richle P, Tietz J, Spohn G, et al. Brolucizumab, a unique single-chain antibody fragment inhibitor of vascular endothelial growth factor. Submitted for publication.

19 Nimz EL, Van't Land CW, Yáñez JA, Chastain JE. Intraocular and systemic pharmacokinetics of brolucizumab (RTH258) in nonhuman primates. The Association for Research in Vision and Ophthalmology (ARVO) Annual Meeting. 2016;57(12):Abstract 4996.

20 Gaudreault J, Gunde T, Floyd HS, Ellis J, Tietz J, Binggeli D, et al. Preclinical pharmacology and safety of ESBA1008, a single-chain antibody fragment, investigated as potential treatment for age related macular degeneration. Invest Ophthalmol Vis Sci. 2012;53(14):3025.

21 Park SJ, Choi Y, Na YM, Hong HK, Park JY, Park KH, et al. Intraocular pharmacokinetics of intravitreal aflibercept (Eylea) in a rabbit model. Invest Ophthalmol Vis Sci. 2016 May; 57(6):2612-7.

22 Gaudreault J, Fei D, Rusit J, Suboc P, Shiu V. Preclinical pharmacokinetics of Ranibizumab
(rhuFabV2) after a single intravitreal administration. Invest Ophthalmol Vis Sci. 2005 Feb;46(2):726-33.

23 Krzystolik MG, Afshari MA, Adamis AP, Gaudreault J, Gragoudas ES, Michaud NA, et al. Prevention of experimental choroidal neovascularization with intravitreal anti-vascular endothelial growth factor antibody fragment. Arch Ophthalmol. 2002 Mar;120(3):338-46.

24 Escher D, Schmidt A, Steiner P, Maurer P, Weissgerber G. Single-chain antibody fragments in ophthalmology. Presented at EURETINA, September 17-20, 2015, Nice, France.

25 Australian Public Assessment Report for Aflibercept, 2014

26 García-Quintanilla L, Luaces-Rodríguez A, Gil-Martínez M, Mondelo-García C, Maroñas $\mathrm{O}$, Mangas-Sanjuan $\mathrm{V}$, et al. Pharmacokinetics of Intravitreal Anti-VEGF Drugs in Age-Related Macular Degeneration. Pharmaceutics. 2019 Jul;11(8):365-87.

27 Fogli S, Del Re M, Rofi E, Posarelli C, Figus M, Danesi R. Clinical pharmacology of intravitreal anti-VEGF drugs. Eye (Lond). 2018 Jun; 32(6):1010-20.

28 Markham A. Brolucizumab: first Approval. Drugs. 2019 Dec;79(18):1997-2000.

29 Nguyen QD, Das A, Do DV, Dugel PU, Gomes A, Holz FG, et al. Brolucizumab: Evo- lution through Preclinical and Clinical Studies and the Implications for the Management of Neovascular Age-Related Macular Degeneration. Ophthalmology. 2020 Jul;127(7): 963-76.

30 Dugel PU, Singh RP, Koh A, Ogura Y, WeissgerberG, Gedif K, et al. HAWK and HARRIER: Ninety-Six-Week Outcomes from the Phase 3 Trials of Brolucizumab for Neovascular Age-Related Macular Degeneration. Ophthalmology. 2020;S0161-6420(20)30570-4.

31 Agostini H, Mulyukov Z, Tsilimbaris M, Calvo $\mathrm{P}$, Bucher F, Gaucher D, et al. Comparison of the efficacy of brolucizumab with natural disease progression in wet AMD using clinical data from the Phase III HAWK and HARRIER trials and modelled placebo data. Curr Eye Res. 2020 Oct;45(10):1298-301.

32 Holz FG, Dugel PU, Chirag DJ, Wykoff CC, Singh RP, Murray C, et al. Visual and expanded anatomical outcomes for brolucizumab versus aflibercept in patients with nAMD: 96week data from HAWK and HARRIER. Presented at the 19th EURETINA Congress, September 5-8, 2019, Paris, France.

33 Baumal CR, Spaide RF, Vajzovic L, Freund $\mathrm{KB}$, Walter SD, John V, et al. Retinal vasculitis and intraocular inflammation after intravitreal injection of brolucizumab. Ophthalmology. 2020 Oct;127(10):1345-59. 